\title{
Development of Portable Device for Monitoring the Lithium Level from Bipolar Disorder Patients.
}

\author{
Jung Ho Kim, Dermot Diamond and King Tong Lau* \\ CLARITY Centre for Sensor Network Technologies, National Centre for Sensor Research \\ Dublin City University \\ Dublin 9, Ireland \\ jung.kim@dcu.ie ,*kim.lau@dcu.ie
}

\begin{abstract}
This research aims at developing low cost portable proactive healthcare technologies to put more control into the hands of patients especially who have mental illness so that the earliest signs of health problems with medications can be detected and corrected. Monitoring prescription drugs such as lithium, clozapine etc is important for safe guarding the well-being of the bipolar sufferers. Therapeutically useful amounts of lithium $(\sim$ 0.6 to $1.2 \mathrm{mmol} / \mathrm{L}$ ) are only slightly lower than toxic amounts $(>1.5 \mathrm{mmol} / \mathrm{L})$, so the concentration of lithium must be carefully monitored during treatment to avoid toxicity. A very sensitive analytical method was proposed for the spectrofluorimetric determination of lithium base on its reaction with 1,4dihydroxyanthraquinone (Quinizarin). The fluorescence is measured at an excitation wavelength of $590 \mathrm{~nm}$ and emission wavelength of $620 \mathrm{~nm}$. Saliva sample was tested using the proposed portable device in order to validate the feasibility of saliva as a sample to detect lithium ions. Calibration results presented that linear range of detection was $0.25 \mathrm{mM} \sim 6.0 \mathrm{mM}$ of $\mathrm{Li}^{+}$in saliva with $\mathrm{R}^{2}=0.99$. The range of detection covers sufficiently the therapeutic range of lithium drugs.
\end{abstract}

Keywords-component; bipolar disorder; lithium; fluorescence; portable device

\section{INTRODUCTION}

According to the American Psychiatric Association (APA) guidelines for treating bipolar disorder, atypical medication for bipolar patients with severe mania or mixed episodes is an antipsychotic medication combined with mood stabilizers, antimanic/depressant agents [1].

Lithium (brand names Eskalith, Lithobid, Lithonate, and Lithotabs) is the most widely used and studied medication for treating bipolar disorder. Lithium helps reduce the severity and frequency of mania. It may also help relieve bipolar depression [2-4]. Although lithium is highly effective at reducing the frequency and intensity of mood swings, it is also a very difficult and potentially dangerous drug for patients. Therapeutically useful amounts of lithium $(\sim 0.6$ to 1.2 $\mathrm{mmol} / \mathrm{L})$ are only slightly lower than toxic amounts $(>1.5$ $\mathrm{mmol} / \mathrm{L}$ ), so the concentration of lithium must be carefully monitored during treatment to avoid toxicity [5]. Also, it is necessary to maintain steady lithium concentration to keep the patient in stable mood. Since lithium's therapeutic dose is uncomfortably close to its toxic dose, meaning it is rather easy to take too much lithium by mistake and become poisoned. Toxically high blood lithium levels can cause respiratory depression, seizures, coma and even death. The monitoring of lithium level for bipolar disorder patients is clearly an important aspect of treatment as the side effects (polyuria, polydipsia, weight gain, cognitive problems, sedation or lethargy) of treatment risks to thyroid and kidney can be reduced or eliminated by dose adjustment or dosage schedule $[5,6]$. Therefore, patients need to have regular tests to monitor the levels of lithium based medication.

Saliva has been considered as an alternative matrix for biochemical parameter monitoring. Human saliva is $98 \%$ water, but it contains many important substances, including electrolytes, mucus, antibacterial compounds and various enzymes. It has been used to assess a variety of disease activities and the levels of certain drugs and hormones [7,8]. Saliva collection is non-invasive and less stressful when compared to blood collection.

This paper presents part of our work in developing a low cost, non-invasive portable healthcare technology to put more control into the hands of patients so that the earliest signs of problems can be detected and corrected by monitoring the drug level from the saliva. Here, a very sensitive analytical method was investigated for the spectrofluorimetric determination of lithium base on its reaction with 1,4-dihydroxyanthraquinone (Quinizarin) $[9,10]$. The fluorescence is measured at $620 \mathrm{~nm}$ with an excitation wavelength of $590 \mathrm{~nm}$.

\section{EXPERIMENTAL}

\section{A. Experimental setup}

A portable biochemical device was developed to measure the intensity of fluorescence. The light source $(\lambda \max : 590 \mathrm{~nm}$, $50 \mathrm{~mW}$ ) and photo detector (OPT101: Texas Instruments) were configured at 90 degree angle in the opaque cuvette holder as shown in Fig. 1. The photo detector was covered by a red band pass filter to detect emission light at $620 \mathrm{~nm}$. Digital output voltage signal from the photo detector was achieved with an 8 bits micro-controller (PIC12F675, Microchip Technology Inc.) and the data were logged and time stamped.

This work is supported by Seventh Framework Programme (FP7) - Grant agreement no. 247777 . 
$B$.

\section{Preparation of reagents and standards}

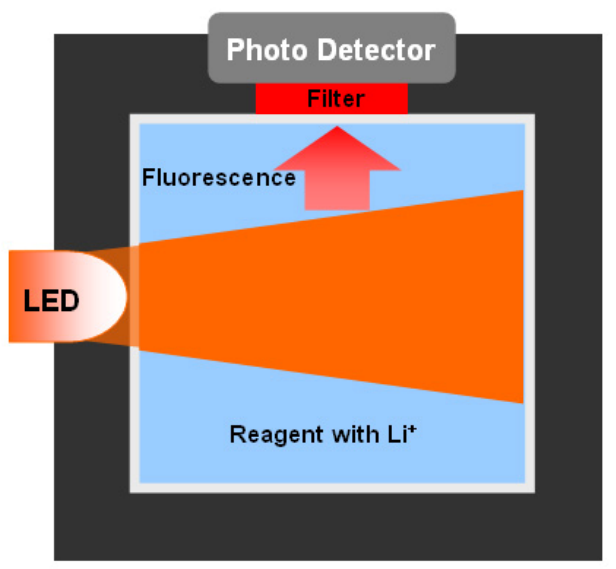

Figure 1. Schematic of the optical sensing module for the measurement of fluorescece.

Analytical grade reagents were used for all experiments and aqueous solutions were prepared using de-ionized water. For the calibration, $50 \mathrm{mM}$ stock lithium solution was prepared by dissolving $\mathrm{LiCl}$ (Sigma Aldrich) in water. Standard solutions of lithium were prepared by diluting the stock solution. $35 \mathrm{mM}$ $\mathrm{NaOH}$ was prepared in water. The fluorescent dye solution was prepared by dissolving 1,4-dihydroxy-anthraquinone (Sigma Aldrich) in dimethylsulfoxide (Sigma Aldrich) to make $15 \mu \mathrm{M}$ stock.

\section{Calibration process for aqueous sample}

$1 \mathrm{~mL}$ of $15 \mathrm{uM}$ quinizarin in dimethylsulfoxide solution was added into a standard quartz or disposable cuvette; followed by $50 \mu \mathrm{l}$ of $0.035 \mathrm{M} \mathrm{NaOH}$ solution. Then $10 \mu \mathrm{L}_{\text {of }} \mathrm{Li}^{+}$sample was spiked into prepared reagent. The mixture was thoroughly stirred before read by the spectrofluorimeter and/or the portable device to measure the intensity of fluorescence.

\section{Calibration process for saliva sample}

Saliva sample was collected from a healthy volunteer. The oral cavity was thoroughly rinsed with water about 5 minutes before to collect newly produced saliva sample. Unstimulated saliva was collected mainly from sublingual glands located under the tongue using dripping method where saliva was allow to drip into a propylene vial. 1-3 $\mathrm{mL}$ of sample was collected each time.

Doped saliva sample was prepared by mixing $35 \mu \mathrm{L}$ of saliva sample with $5 \mu \mathrm{L}$ of $\mathrm{Li}^{+}$solution with known concentration. The doped saliva was added into $1 \mathrm{~mL}$ of $15 \mathrm{uM}$ quinizarin in DMSO with $8 \mu \mathrm{L}$ of $0.28 \mathrm{M} \mathrm{NaOH}$. After mixing the sample and reagent, the fluorescence was measured by spectrofluorimeter and the in-house developed portable device.

\section{RESULTS}

Excitation and emission spectra scan was performed to investigate the optimal wave length for the detection of lithium. and scanned results showed in Fig. 2. Two major excitation

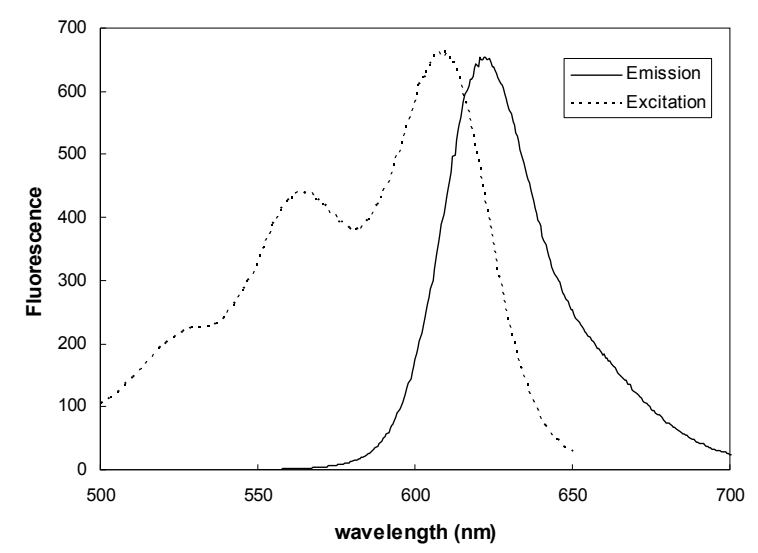

Figure 2. The excitation scan and emission scan of $15 \mu \mathrm{M}$ quinizarin in the presence of $25 \mu \mathrm{M}$ lithium.

peaks were found at $565 \mathrm{~nm}$ and $610 \mathrm{~nm}$. Emission peak was found at $624 \mathrm{~nm}$. Ideally excitation at $610 \mathrm{~nm}$ should be used to give stronger fluorescence intensity but due to the difficulty in filtering out the excitation wave length, LED with peak emission at $590 \mathrm{~nm}$ was selected as excitation light source.

The optimization of reagent was performed in order to increase the sensitivity and stability of proposed spectrofluorimetric method. The result of optimization presented in Fig. 3 that the highest intensity of fluorescence was measured with the concentration of $15 \mu \mathrm{M}$ quinizarin in DMSO. Higher concentration of quinizarin did not give more emission due to re-absorption of emission light by the dye. $40 \mu \mathrm{M}$ of $\mathrm{NaOH}$ was used as insufficient amount of $\mathrm{NaOH}$ caused fluorescence instability.

The calibration (3 repeats) was performed with aqueous $\mathrm{Li}^{+}$ standards. The calibration curve is linear over the concentration ranges of $0-40 \mu \mathrm{M}$ of Lithium ion with $\mathrm{R}^{2}=0.996$ as shown in Fig. 4. In comparison with standard spectrofluorimeter, the linearity range of portable device showed superior performance with much lower detection limit, hence it is an excellent

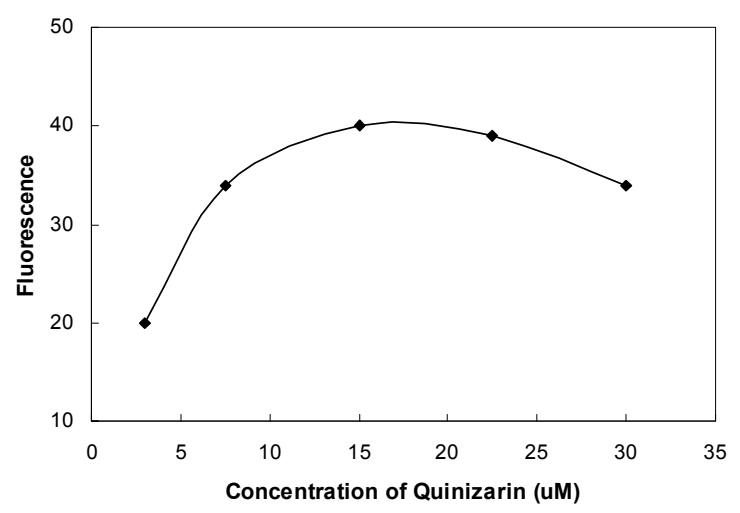

Figure 3. Optimization of dye (quinizarin) concentration in DMSO. 

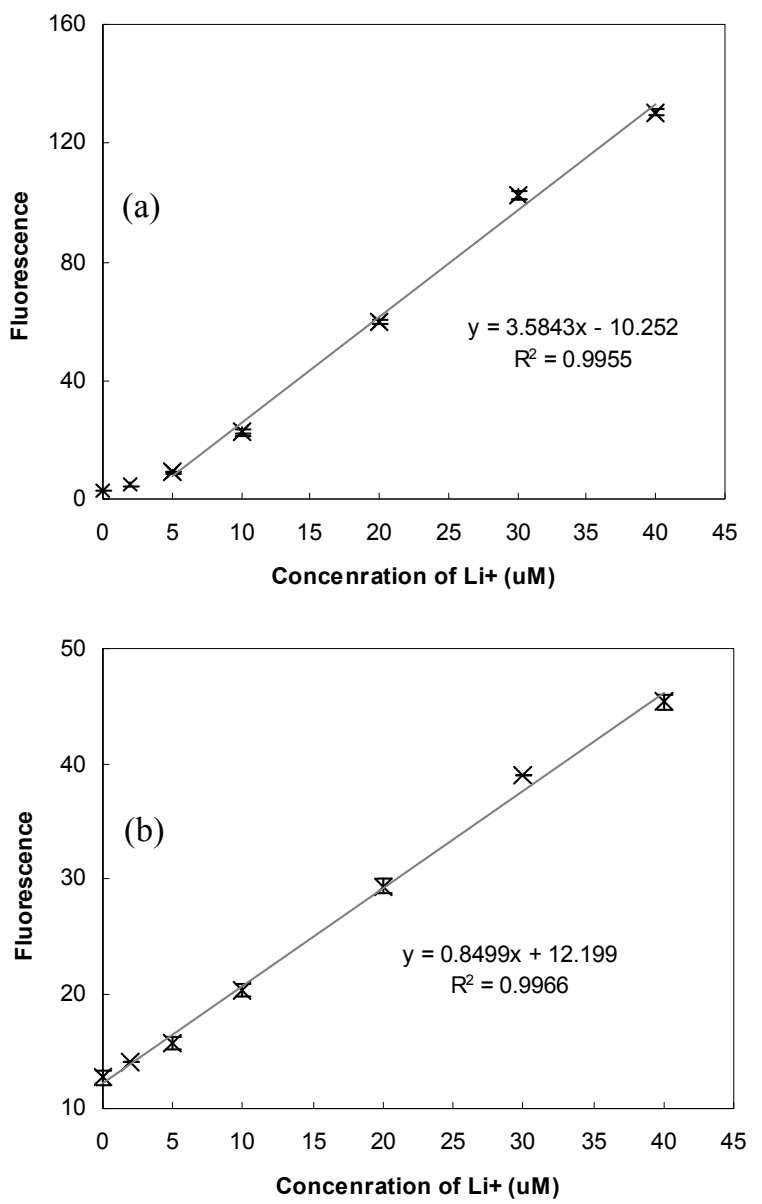

Figure 4. Validation of developed portable device with spectrofluorimeter. The measurement of fluorescece using (a) spectrofluorimeter (b) portable device.

sensing device for lithium ions detection.

Saliva sample was tested using portable device in order to validate the feasibility of saliva as a sample to detect lithium ions. According to the clinical tests, the salivary lithium concentration is $2.2 \sim 2.5$ times higher than serum lithium with significant correlation factor [11-13]. Unstimulated saliva was collected and known concentration of $\mathrm{Li}^{+}$was spiked into the saliva to prepare the $\mathrm{Li}^{+}$doped saliva. Using lithium doped salivary sample, calibration was performed. Results presented in Fig. 5 show calibrations obtained from different saliva samples collected on different days. Similar results were obtained with linear range of detection between $0.25 \mathrm{mM} \sim 6.0$ $\mathrm{mM}$ of $\mathrm{Li}^{+}$and $\mathrm{R}^{2}=0.990$. When we consider the therapeutic range of lithium in serum is $0.6 \sim 1.0 \mathrm{mM}$, the expected salivary lithium concentration would be higher than lithium in serum. Linear range of the proposed method covers sufficiently the therapeutic range of lithium drugs.

\section{CONCLUSIONS}

Saliva sample was tested using portable device in order to validate the feasibility of saliva as a sample to detect lithium

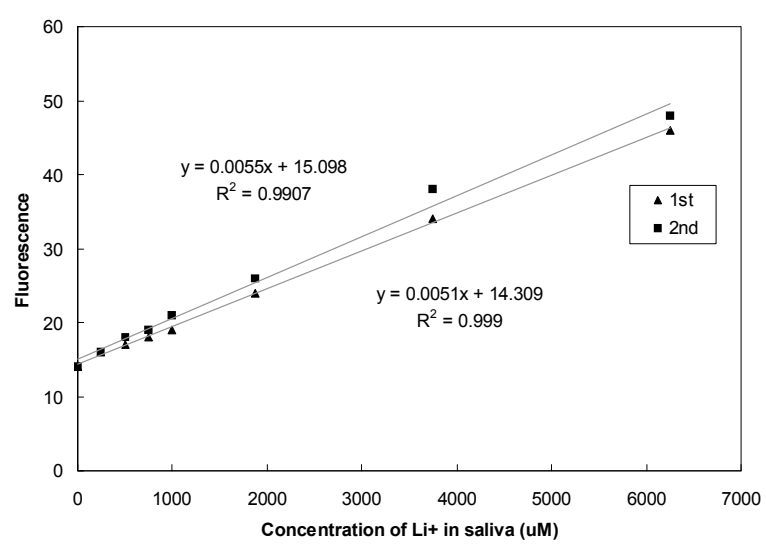

Figure 5. Calibration results from $\mathrm{Li}^{+}$doped saliva with portable device.

ions. Calibration results presented that the linear range of detection was $0.25 \sim 6.0 \mathrm{mM}$ of $\mathrm{Li}^{+}$in saliva with $\mathrm{R}^{2}=0.990$. The range of detection offered by the portable device covers sufficiently the therapeutic range of lithium. This rapid and simple way of lithium measurement will help to monitor and thus maintain a steady lithium concentration.

\section{ACKNOWLEDGMENT}

This work is supported by Seventh Framework Programme (FP7) - Grant agreement no. 247777.

\section{REFERENCES}

[1] K. N. Fountoulakis, E. Vieta, J. Sanchez-Moreno, S. G. Kaprinis, J. M. Goikolea and G. S. Kaprinis, "Special review article: Treatment guidelines for bipolar disorder: A critical review," Journal of Affective Disorders, Vol. 86(1), pp. 1-10, 2005.

[2] M. E. Thase, and G. S. Sachs, "Bipolar Depression: Pharmacotherapy and Related Therapeutic Strategies," Biological Psychiatry, Vol. 48(6), pp. 558-572, 2000.

[3] G. S. Sachs and M. E. Thase, "Therapeutic approach - Bipolar disorder therapeutics: maintenance treatment," Vol. 48(6), pp. 573-581, 2000.

[4] R. Williams and A. J. Harwood, "Lithium therapy and signal transduction," Trends in Pharmacological Sciences, Vol. 21(2), pp. 6164, 2000.

[5] M. E. G. Sansone and D. K. Zeigler, " Lithium Toxicity: A Review of Neurologic Complications," Clinical Neuropharmacology, Vol. 8(3), pp. 242-248, 1985.

[6] N. J. Delva and E. R. Hawken, "Preventing lithium intoxication - Guide for physicians," Canadian Family Physician, vol. 47, pp. 1595-1600, 2001.

[7] D. B. Ferguson, "Current diagnostic uses of saliva," Journal of dental research, Vol. 66(2), pp. 420-424, 1987.

[8] C. F. Streckfus, L. R. Bigler, "Saliva as a diagnostic fluid," Journal of oral diseases, Vol. 8(2), pp. 69-76, 2002.

[9] L. C. Rodriguez, C. J. Linares and M. R. Ceba, "Solvent donicity influence in the fluorescence emission of the lithium/1,4dihydroxyanthraquinone system. Improvement in the quantification of lithium," Analytical Letters, vol. 30(4), pp. 847-866, Feb. 1997.

[10] M. R. Ceba, A. F. Gutierrez and C. M. Sanchez, "Some observations on the use of 1,4-dihydroxyanthraquinone as a fluorometric reagent for 
traces of lithium," Microchemical Journal, Vol. 32(3), pp. 286-292, 1985.

[11] A. Verghese, N. Indrani, K. Kuruvilla and P. Hill, "Usefulness of Saliva Lithium Estimation," The British Journal of Psychiatry, Vol. 130, pp. 148-150, 1977

[12] H. Ben-Aryeh, H. Naon, R. Szargel, D. Gutman and A. Hefetz, "Salivary lithium concentration - A tool for monitoring psychiatric patients," J.
Oral Surgery, Oral Medicine, Oral Pathology, Vol. 50, pp. 127-129, 1980.

[13] R. Perry, M. Campbell and L. Anderson, "Saliva lithium levels in children: their use in monitoring serum lithium levels and lithium side effects.” J. Clin. Psychopharmacol., Vol. 4, pp. 199-202, 1984. 